\title{
Effect of intranasal administration of Semliki Forest virus recombinant particles expressing interferon- $\beta$ on the progression of experimental autoimmune encephalomyelitis
}

\author{
K. QUINN ${ }^{1,3}$, S.E. GALBRAITH ${ }^{1,2,4}$, B.J. SHEAHAN ${ }^{2}$ and G.J. ATKINS ${ }^{1}$ \\ ${ }^{1}$ Virus Group, Moyne Institute of Preventive Medicine, School of Genetics and Microbiology, Trinity College, Dublin 2; \\ ${ }^{2}$ Veterinary Sciences Centre, School of Agriculture, Food Science and Veterinary Medicine, University College Dublin, \\ Belfield, Dublin 4, Ireland; ${ }^{3}$ Molecular Physiology and Therapeutics Branch, National Institute of Dental and \\ Craniofacial Research, National Institutes of Health, 10 Center Drive, Bethesda, MD 20892; \\ ${ }^{4}$ Department of Pathology, University of Texas Medical Branch, 301 University Blvd., \\ Galveston, TX 77555-0609, USA
}

Received February 5, 2008; Accepted March 3, 2008

\begin{abstract}
The effect of intranasal (IN) administration of Semliki Forest virus (SFV) recombinant particles expressing interferon- $\beta[$ IFN- $\beta$, a partially effective treatment for multiple sclerosis (MS)] on the progression of experimental autoimmune encephalomyelitis (EAE, a murine model for MS) was investigated. The murine IFN- $\beta$ gene was cloned from SFV-infected mouse brain by RT-PCR into an SFV-enhanced expression vector, pSFV10-E, from which IFN-B-expressing recombinant particles (rSFV10-E-IFN- 3 ) were prepared. Expression studies using immunohistochemistry and viral inhibition assay in BHK and murine L929 cells confirmed increased expression of IFN-B. High level expression in the central nervous system (CNS) following IN inoculation was confirmed by the excision of olfactory bulbs, brain and spinal cord, and the detection of IFN- $\beta$ levels in homogenised tissue by ELISA. rSFV10-E-IFN- $\beta$ particles were administered IN to $\mathrm{C} 57 / \mathrm{B} 16$ mice that had been induced for EAE using the encephalogenic peptide myelin oligodendrocyte glycoprotein (MOG) 35-55. The progression of EAE was measured by clinical score, weight loss and pathology. As previously shown, treatment with empty rSFV10-E particles moderately exacerbated EAE, as did continuous treatment with rSFV10E-IFN- $\beta$ particles. Inhibition of disease with rSFV10-EIFN- $\beta$ particles was dependent on the number and timing of treatments. Fewer treatments, administered before the effector stage, led to an improvement in clinical and pathology score.
\end{abstract}

Correspondence to: Professor Gregory J. Atkins, Virus Group, Moyne Institute of Preventive Medicine, School of Genetics and Microbiology, Trinity College, Dublin 2, Ireland

E-mail: gatkins@tcd.ie

Key words: ß-interferon, Semliki Forest virus vector, experimental autoimmune encephalomyelitis, demyelination
In conclusion, the timing and frequency of IN administration of rSFV10-E-IFN- $\beta$ particles are critical to disease outcome, with treatment prior to the effector stage being most effective.

\section{Introduction}

Semliki Forest virus (SFV) is a positive-strand enveloped RNA virus that has been developed into a prototype vector for vaccine construction and cancer therapy $(1,2)$. Due to its ability to penetrate the central nervous system (CNS) following both intranasal (IN) and peripheral inoculation, SFV has also been developed as a gene therapy agent for the CNS (3-6). After IN inoculation, the virus reaches the CNS via the olfactory bulb. Once there, it infects neurons and oligodendrocytes and travels through the limbic pathways to further infect other areas of the brain $(7,8)$.

The SFV split helper system, based on the SFV4 strain of SFV, has also been employed as a means of therapeutic delivery to the CNS. In this system, the viral structural gene open reading frame is replaced with a multiple cloning site (MCS) to allow insertion and expression of a foreign gene. Structural genes are supplied in trans from two separate helper vectors, the helper spike and the helper capsid. On cotransfection of all three vectors into BHK cells, defective recombinant particles, capable of one round of virus multiplication only upon infection of susceptible cells, are produced and released from the cells. In the process, the cloned foreign gene is expressed transiently but at a high level (9).

Administration of recombinant SFV particles expressing the reporter gene EGFP showed that high level EGFP expression could be detected in incoming axons from receptor cells in the olfactory epithelium, which terminate in the glomerular area of the olfactory bulbs. Unlike previous studies with this and other vectors, which have used the invasive IC route $(10-12)$, the IN route is non-traumatic. Expression occurred in the olfactory bulb as early as 1 day after IN inoculation, and continued for up to 10 days with no detectable damage. Vector RNA was detected in the nasal passage of infected animals 
only, indicating that the viral vector does not enter the CNS. These findings also suggest that transcription and/or replication of vector RNA occurs in the cells of the olfactory mucosa, which have the capability to regenerate, while only the recombinant protein enters the CNS. Similarly high expression of a cloned cytokine, IL-10, was shown in the nasal mucosa and olfactory bulbs of mice for up to 7 days after IN inoculation with rSFV-IL-10 vector particles. It is therefore probable that, following IN administration of recombinant particles, only the protein product of the cloned gene enters the CNS (5).

Experimental autoimmune encephalomyelitis (EAE) is a well-characterised animal model of human multiple sclerosis (MS). Like MS, it is an inflammatory demyelinating disease of the CNS. A classical $\mathrm{CD}^{+}$autoimmune disease, EAE is caused by myelin-specific $\mathrm{CD} 4^{+} \mathrm{T}$ helper (Th1) cells infiltrating the brain and attacking the myelin sheath $(13,14)$. The other CD4 ${ }^{+}$MHC class II restricted cells, Th2 cells, are associated with disease recovery (13-17). The type of EAE induced is dependent on the immunization protocol, the animal strain and the antigen used. Myelin oligodendrocyte glycoprotein (MOG)-induced EAE, utilised in this study, consists of an initial induction phase, an effector phase at which T-cell response to the encephalitogenic peptide has fully developed but T-cell accumulation in the CNS has not yet occurred (days 12-17 in this study) (5) and a recovery phase (14).

Previous research has concentrated on the treatment of EAE using immunomodulators such as cytokines $(18,19)$, or used plasmid-based DNA vectors and viral vectors expressing cytokines $(10,11,20,21)$. We used a recombinant SFV vector expressing IL-10 to treat EAE induced in C57/B16 mice with MOG 35-55. The rSFV-IL-10 vector was shown to significantly reduce disease severity and retard the onset of disease, as shown by improved clinical score and weight gain. In addition, milder and less frequent brain and spinal cord lesions were observed in the rSFV-IL-10-treated animals (5).

In this study, we utilised the same SFV particle vector system to express interferon- $\beta$ (IFN- $\beta$ ), a cytokine known to be partially effective in the treatment of relapsing remitting MS. The vector used, rSFV10-E, shows increased protein production due to the presence of a capsid translational enhancer region adjacent to the MCS $(9,22,23)$. This capsid enhancer region consists of 34 amino acids of the SFV capsid protein followed by an FMDV2A peptide. The enhancer region increases expression of the cloned gene, and the FMDV peptide is responsible for cleaving the foreign protein from the capsid enhancer. We previously used this vector to express IL-12 in the treatment of metastasizing cancer in mice (23).

Previous studies have shown that successful treatment of EAE and indeed MS with IFN- $\beta$ is time and dose dependent. When the long-term dose received by MS patients was reduced from $250 \mu \mathrm{g}$ every $48 \mathrm{~h}$ to $30 \mu \mathrm{g}$ every week, only $23 \%$ remained relapse-free compared to $79 \%$ at the higher dose and frequency (24). Similarly, SC administration of rIFN- 3 dose dependently inhibited EAE in Lewis rats, provided the treatment was continued for three weeks (25). A previous study utilised a retroviral viral vector expressing IFN- $\beta$ to successfully treat EAE induced in Biozzi ABH mice by injection of spinal cord homogenate. In this study, the vector was encapsulated in a DNA-liposome complex and administered by IC inoculation (26).
A previous study showed that IN administration of IFN- $\beta$ to non-EAE rats was capable of bypassing the blood-brain barrier to target the CNS and cervical lymph nodes, suggesting a non-invasive treatment strategy for EAE and MS (27). The present study involved IN administration of an IFN-Bexpressing SFV recombinant particle vector to mice, a technique based on our previous study for IL-10 (5). Although treatments were administered during the induction, effector and recovery stages of EAE, it was found that only those treatments given immediately before the effector period postponed disease progression.

\section{Materials and methods}

SFV expression vectors. SFV expression vectors pSFV1 and pSFV10-E and split-helper vectors pSFV-Helper S2 and pSFV-Helper CS219A were a gift from Professor P. Liljeström, Karolinska Institute, Sweden. Plasmids were transformed and grown in E. coli DH5 $\alpha$ cells and extracted using the Qiagen Mini Prep Kit (Qiagen, UK).

Cell culture. The BHK-21 cell line was obtained from the American Type Culture Collection (ATCC). The sBHK cell line was a gift from Professor P. Liljeström. Both cell lines were grown in BHK medium supplemented with $5 \%(\mathrm{v} / \mathrm{v})$ fetal bovine serum (FBS), 5\% (v/v) tryptose phosphate broth, $2 \%(\mathrm{v} / \mathrm{v}) 1 \mathrm{M}$ HEPES and $1 \%(\mathrm{v} / \mathrm{v})$ penicillin-streptomycinL-glutamine. The murine cell line L929 was obtained from the European Collection of Cell Cultures (ECACC) and was grown in DMEM supplemented with $10 \%(\mathrm{v} / \mathrm{v})$ FBS and $1 \%$ (v/v) penicillin-streptomycin-L-glutamine.

Animals and virus strains. Specific pathogen-free female 40to 60-day-old BALB/c and C57/B16 mice were obtained from the BioResources Unit, Trinity College Dublin and Harlan (Bicester, UK) respectively, and maintained under pathogenfree conditions with food and water provided in accordance with the principles set down in SI 17/94 of the European Union. A working stock of the avirulent A7 strain of SFV was propagated in BHK-21 cells from plaque-purified seed stock and titrated by plaque assay on BHK-21 cells. For virus infection, BALB/c mice were inoculated IP with $10^{6}$ plaqueforming units of A7 virus in $500 \mu 1$ phosphate-buffered saline (PBS). Mice were anesthetised with halothane, and brains and spleens were removed at days 2, 4 and 7 post infection.

Amplification and cloning of the IFN- $\beta$ gene. Total-RNA was isolated from the brains and spleens of SFV A7-infected mice, and RT-PCRs were performed as previously described (5). The following primers, incorporating an $\mathrm{XmaI}$ restriction site (bold) and a ribosomal binding site (underlined), were designed to amplify and clone murine IFN- $\beta$ (GenBank sequence) from cDNA as pSFV1 lacks a Kozak element: murine IFN-B F 5'-AATCCCGGGGCACCATGAACAACAGGTGGA-3' and murine IFN- $\beta$ R 5'-GCGCCCGGGTCTTCAGTTTTG GAAGTTTCT-3'. Negative control reaction mixes using PBS mock-infected brains were included in each RT-PCR reaction set. Internal controls for the reaction included mixes lacking RNA or the RT enzyme. RT-PCR products were purified using 
the Qiagen PCR purification protocol. Purified IFN- $\beta$ product and cloning vector pSFV1 were cut at their unique $\mathrm{XmaI}$ restriction site (NEB). Digested IFN- $\beta$ was pooled and gel extracted using Qiagen Gel Extraction Kit, and digested pSFV1 was cleaned with a Qiagen Nucleotide Removal Kit. Linearised pSFV1 and digested product were mixed at a ratio of 1:10 in a $20-\mu 1$ ligation mixture containing 1 unit Quick T4 Ligase and 10X ligase buffer (NEB), and incubated for $5 \mathrm{~min}$ at room temperature. This ligation mixture was transformed into $E$. coli $\mathrm{DH} 5 \alpha$ cells, and colonies were screened by digestion with the $X m a I$ restriction enzyme. Positive clones were confirmed by sequencing. For pSFV10-E cloning, the IFN- $\beta$ gene was amplified from the rSFV1-IFN- $\beta$ plasmid using a new forward primer lacking the Kozak element, 5'-AATCCCGGGATG AACAACAGGTGGA-3' and the same reverse primer, and then ligated with $X m a I$ linearised pSFV10-E as described above, but overnight using T4 high efficiency DNA ligase and buffer (Promega). Positive clones were confirmed using the same sequencing primers.

$I F N-\beta$ expression. Expression of IFN- $\beta$ from the rSFV10-EIFN- $\beta$ vector was detected by electroporating RNA from $1.5 \mu \mathrm{g}$ of in vitro transcribed linearised plasmid into BHK-21 cells (BioRad gene pulser) as previously described $(5,9)$. The rSFV10-E-IFN-B plasmid was linearised with the NruI restriction enzyme. Linearised empty SFV plasmids were in vitro transcribed and electroporated into BHK-21 cells as negative controls. PBS mock electroporated cells were also included. Following electroporation, cells were grown on $22 \times 22 \mathrm{~mm}$ coverslips in a 6-well plate for $18-20 \mathrm{~h}$ at $37^{\circ} \mathrm{C}$. After fixation in $4 \%$ paraformaldehyde and permeabilization in acetic acid:ethanol $(1: 2)$ at $-20^{\circ} \mathrm{C}$, endogenous peroxidase activity was blocked with $3 \%$ (vol/vol) $\mathrm{H}_{2} \mathrm{O}_{2}$ in PBS. Nonspecific binding sites were blocked with $10 \%(\mathrm{v} / \mathrm{v})$ normal goat serum (NGS) in PBS for 20 min at $37^{\circ} \mathrm{C}$. To detect IFN-B, cells were incubated with rabbit anti-mouse IFN- $\beta$ polyclonal antibody (1:100 dilution, $\mathrm{PBL}, \mathrm{UK})$ for $1 \mathrm{~h}$ at $37^{\circ} \mathrm{C}$, then with biotinylated secondary antibody (1:200 dilution in NGS blocking buffer) for $30 \mathrm{~min}$ at RT, and finally with avidinbiotin-peroxidase complex (Vectastain Elite ABC Reagent) for $30 \mathrm{~min}$ at room temperature. Staining was visualised using diaminobenzidine chromagen (Sigma), and the cells were counterstained with Harris haematoxylin $(\mathrm{BDH})$ and ammonia water.

rSFV particle production and titration. rSFV10-E-IFN- 3 or empty particle controls were prepared as previously described $(5,23)$. Briefly, sBHK cells were co-transfected by electroporation with rSFV RNA and the helper capsid and envelope RNAs, and cells were incubated at $33^{\circ} \mathrm{C}$ in $5 \% \mathrm{CO}_{2}$ for $36-40 \mathrm{~h}$. After incubation, the cell culture medium was clarified $3 \mathrm{x}$ by centrifugation at $4^{\circ} \mathrm{C}$ in a Beckman SW34 rotor for $15 \mathrm{~min}$ at $18,000 \mathrm{rpm}$. Particles were concentrated by ultracentrifugation through a $20 \%(\mathrm{w} / \mathrm{v})$ sucrose cushion in a Beckman SW28 rotor at $25,000 \mathrm{rpm}$ for $2 \mathrm{~h}$ at $4^{\circ} \mathrm{C}$. Virus pellets were re-suspended in TNE buffer $(50 \mathrm{mM}$ Tris- $\mathrm{HCl}$ pH 7.4, $100 \mathrm{mM} \mathrm{NaCl}, 0.1 \mathrm{mM}$ EDTA), and aliquots were snap frozen in liquid $\mathrm{N}_{2}$ and stored at $-70^{\circ} \mathrm{C}$ until use. To determine the titer of the stocks, sBHK cells were grown on $22 \times 22 \mathrm{~mm}$ coverslips in 6-well plates, infected with serial dilutions of viral particles and monitored for expression of IFN- $\beta$ by indirect immunofluorescence; rSFV10-E-IFN- $\beta$ particles were titrated with rabbit anti-mouse IFN- $\beta$ antibody. Empty particles were titrated in the same way using an antibody specific for the nsP4 protein of SFV.

Viral inhibition assay. This assay was carried out to confirm the biological activity in cell culture of the IFN- $\beta$ expressed by rSFV10-E-IFN-ß particles. BHK-21 and murine L929 cells were infected with rSFV10-E-IFN- $\beta$ particles and rSFV10-E empty particles at a multiplicity of infection (MOI) of 1, 10 and 100 , and incubated at $37^{\circ} \mathrm{C}$ in $5 \% \mathrm{CO}_{2}$ for $24 \mathrm{~h}$. Supernatants were then centrifuged at $14,000 \mathrm{rpm}$ for $5 \mathrm{~min}$ at $4^{\circ} \mathrm{C}$ to remove cell debris, aliquoted and frozen at $-70^{\circ} \mathrm{C}$ for subsequent assay for IFN-ß. L929 cells were seeded in 96-well plates at $3 \times 10^{4}$ cells/well and grown overnight at $37^{\circ} \mathrm{C}$. Standard IFN- $\beta$ (1x10 $10^{6} \mathrm{U}$ of activity/ml; PBL, UK) was serially 10 -fold diluted in DMEM, and $50 \mu 1$ of each dilution was added to duplicate wells at a starting concentration no greater than $50 \mathrm{U} /$ well. Samples were serially diluted 2 -fold, and $50 \mu 1$ was added to each well. The plate was incubated at $37^{\circ} \mathrm{C}$ for $18-24 \mathrm{~h}$, then the samples were removed and the cells infected with SFV A7 $(\mathrm{MOI}=1)$ for $1 \mathrm{~h}$. The virus inoculum was removed, fresh medium was added and the plate was incubated for $48 \mathrm{~h}$. CellTiter 96 Reagent $(10 \mu \mathrm{l})$ containing MTS tetrazolium compound (Promega) was added to each well, and the plate was further incubated for 2-3 h. Absorbance was read at $492 \mathrm{~nm}$ on a Multiskan RC plate reader. Controls included infected untreated cells and mock-infected treated cells.

Expression in mice. Three groups of 9 C57/B16 mice were inoculated IN with $10^{7}$ IU of rSFV10-E-IFN- 3 and rSFV10-E particles in $20 \mu 1 \mathrm{TNE}$ and with TNE alone by the placement of a droplet on each nostril. Three mice from each group were sampled 24, 48 and $72 \mathrm{~h}$ post inoculation, and their olfactory bulbs, brains and spinal cords were removed, pooled and homogenised in $2 \mathrm{ml}$ of Tris- $\mathrm{HCl} \mathrm{pH} 8.0$ buffer. Supernatants were clarified by centrifugation $\left(3500 \mathrm{rpm}, 10 \mathrm{~min}, 4^{\circ} \mathrm{C}\right)$ and stored at $-70^{\circ} \mathrm{C}$. IFN- $\beta$ levels were measured by capture ELISA (PBL, USA) according to the manufacturer's protocol. The total protein concentration of each sample was measured using Bradford Reagent (Sigma) to express cytokine levels/mg of total protein. Two way ANOVA and Bonferroni post-tests were used for statistical comparison between treatment groups.

EAE induction, clinical assessment and treatment with rSFV10-E-IFN- $\beta$ particles. Disease was induced in C57/B16 mice using the encephalitogenic peptide MOG 35-55 (MEV GWYRSPFSRVVHLYRNGK, Genemed Synthesis, USA) as previously described (5). From the first day of disease onset (day 8/9 post immunization) animals were weighed and graded using the following neurological scale: 0 , no clinical sign; 1 , limp tail and feet; 2, impaired righting reflex; 3, partial hind limb paralysis; 4, complete hind limb paralysis; 5 , moribund; 6 , death. Animals were scored 0.5 less than the indicated grade when they exhibited signs less than the maximum severity. Progression of the disease was expressed as the mean daily EAE score for each group after induction of the disease. Due to ethical restrictions, the animals were not allowed to relapse; 


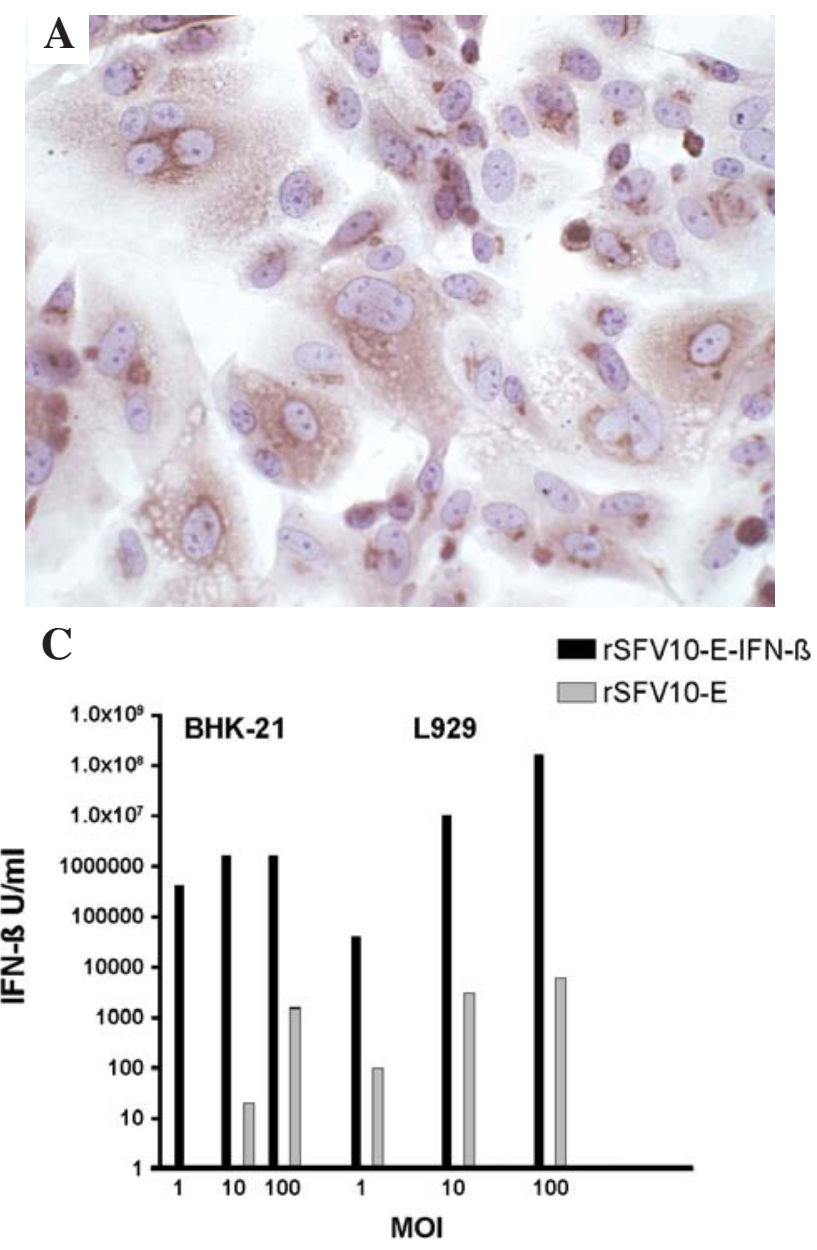

Figure 1. Expression of IFN- $\beta$ from the rSFV10-E-IFN- $\beta$ vector in BHK-21 and $\mathrm{L} 929$ cells. BHK-21 cells were electroporated with RNA in vitro transcribed from linearised rSFV10-E-IFN- $\beta$ particle vector (A) and PBS (B). Cells were fixed and stained for the presence of IFN- $\beta$ after $24 \mathrm{~h}$. (C) BHK-21 and murine L929 cells were infected with rSFV10-E-IFN- $\beta$ and rSFV10-E empty particle controls at MOIs of 1,10 and 100, and mock-infected with buffer alone. Supernatant samples were harvested and assayed for the presence of IFN- $\beta$ after a 24-h incubation. Note the log scale on the y-axis; bars represent triplicates of a single experiment. Two way ANOVA and Bonferroni post-tests were used for statistical comparison between treatment groups. No IFN- $\beta$ was detected in the buffer-treated cells.

therefore, the disease was monitored for up to 30 days only. In all treatments with rSFV10-E particles, animals received $10^{7} \mathrm{IU}$ of rSFV particles in $20 \mu 1 \mathrm{TNE}$ IN. A droplet placed on the end of each nostril was inhaled by the animal.

In the continuous treatment experiment, EAE was induced in a total of $42 \mathrm{C} 57 / \mathrm{B} 16$ mice. The animals were divided into three groups of 14 , and were treated with rSFV10-E-IFN- $\beta$ particles, rSFV10-E particles or TNE buffer alone on days 3 , $6,9,12,15,18,21,24$ and 27 post disease induction (PDI). In both effector stage experiments, EAE was induced in 30 animals; three groups of 10 received five rSFV10-E-IFN- $\beta$, rSFV10-E and TNE treatments on days 5, 8, 11, 13 and 17 in one experiment and four treatments on days 12, 15, 16 and 17 in another. In the pre-effector stage treatments, EAE was induced in 30 animals; three groups of 10 received four IN treatments on days 5, 8,11 and 12 PDI. This experiment was repeated using three groups of 12 animals, 36 in total. Statistical analysis of the different treatment groups was carried out using the Mann-Whitney non-parametric ranking test (5).

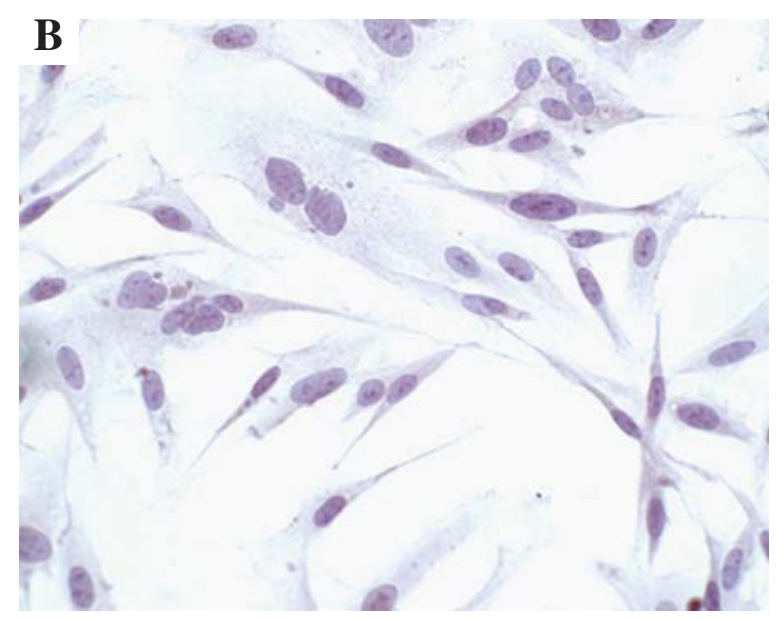

Histological studies. For the continuous treatment experiment, two mice from each group were sacrificed at day 28 PDI. Mice were anesthetised with halothane and perfused via the left ventricle with PBS followed by $10 \%$ formal saline for $5 \mathrm{~min}$. Carcasses were left in fixative overnight prior to removal of brains and spinal cords. Fixed tissues were processed in paraffin wax, and 4- $\mu \mathrm{m}$ sections were stained with hematoxylin and eosin (H\&E). Brains and spinal cords were examined in a blinded manner and assigned an estimated score for the parameters of EAE (i.e. myelin vacuolation, axonal degeneration, leukocytic infiltration and glial activation). The severity and distribution of lesions were evaluated using a five-point grading system. Grade 1 lesions were small and isolated in the spinal cord; grade 5 lesions were diffuse and involved brain stem areas and the spinal cord. From the experiment involving pre-effector phase treatment, 3 mice from each of the groups were sacrificed at day 22 PDI. Tissues were processed in the same fashion and graded according to the same parameters as above. In the repeat experiment, 3 mice from each group were sacrificed and perfused at day 15 . Tissues were processed and lesions graded as above.

\section{Results}

IFN- $\beta$ expression. The IFN- $\beta$ gene was amplified from mouse brain RNA at day 2 post infection (DPI) and cloned into the pSFV vector. Positive clones were confirmed by restriction digestion and sequencing, and IFN- $\beta$ expression by immunohistochemical staining of BHK-21 cells electroporated with transcribed RNA. Diffuse brown cytoplasmic staining was evident at $24 \mathrm{~h}$ post electroporation (Fig. 1A). No staining was evident in the control cells electroporated with TNE buffer (Fig. 1B) or rSFV10-E empty particles (not shown). Infection of BHK and L929 cells with rSFV10-E empty particles produced low levels of IFN- $\beta$ at MOIs of 10 and 100 compared to rSFV10-IFN- $\beta$ particles, which increased expression 10,000 -fold $(\mathrm{p}<0.01)$. L929 cells showed greater IFN- $\beta$ expression at these MOIs than BHK-21 cells, with levels reaching $10^{8} \mathrm{U} / \mathrm{ml}$ (Fig. 1C, note that scale is logarithmic). Mock-infected cells produced no IFN- $\beta$.

To examine IFN- $\beta$ expression in mice, animals were inoculated IN with rSFV10-E empty particles, rSFV10-EIFN- $\beta$ particles and TNE buffer alone. After 24,48 and $72 \mathrm{~h}$, 


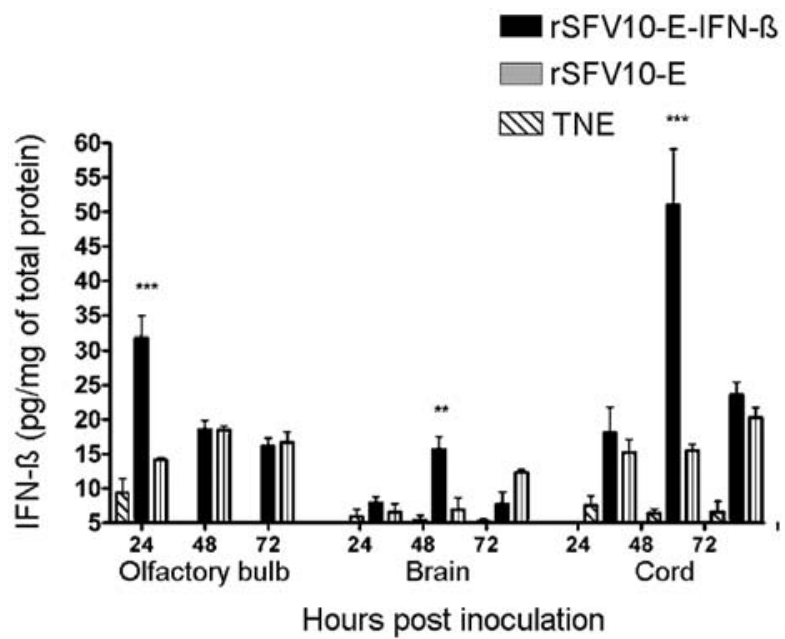

Figure 2. Expression of IFN- $\beta$ in the CNS following intranasal inoculation with rSFV10-E-IFN- $\beta$ particles. Three groups of 9 C57/B16 mice were inoculated IN with rSFV10-E-IFN-B particles, rSFV10-E empty particles and TNE buffer alone, and sacrificed 24,48 and $72 \mathrm{~h}$ after infection. The olfactory bulbs, brain and spinal cord were isolated, each tissue was pooled and homogenised, and levels of IFN- $\beta$ were measured by ELISA and expressed as $\mathrm{pg} / \mathrm{mg}$ of total protein. Each pg of IFN-B equals 12 units of specific activity. Two way ANOVA and Bonferroni post-tests were used for statistical comparison between treatment groups.

the level of IFN- $\beta$ in the olfactory bulbs, brains and spinal cords was measured using a mouse IFN- $B$ ELISA kit. TNEand rSFV10-E particle-inoculated mice produced low levels of IFN- $\beta$ in all tissues. Mice receiving rSFV10-E-IFN- $\beta$ particles expressed significantly more IFN- $\beta$ in the olfactory bulbs at $24 \mathrm{~h}$ post inoculation than did TNE- and rSFV10-E particle- inoculated mice (Fig. 2, p<0.001). After 48 h, significantly more IFN- $\beta$ was present in the brains of rSFV10-E-IFN- $\beta$ inoculated mice than in the brains of TNE- and rSFV10-E particle-inoculated mice $(\mathrm{p}<0.01)$. A significant increase was also observed in the level of IFN- $\beta$ present in the spinal cord of rSFV10-E-IFN- $\beta$-inoculated mice at $48 \mathrm{~h}$ post inoculation $(\mathrm{p}<0.001)$, with levels reaching $60 \mathrm{pg} / \mathrm{mg}$ of total protein. By $72 \mathrm{~h}$ post inoculation, IFN- $\beta$ levels in the tissues from rSFV10E-IFN-B-inoculated mice had dropped to the same levels as in the tissues from rSFV10-E particle- and TNE-inoculated mice.

Effect of treatment on the course of EAE. After EAE induction, mice were treated IN with IFN-ß-expressing particles, rSFV10-E empty particles and TNE buffer. Disease progression was monitored by clinical score and weight loss. The experiments carried out to optimise treatment are summarised in Table I. Continuous treatment (days 3, 6, 9, 12, 15, 18, 21, $24,27)$ with rSFV10-E-IFN- $\beta$ particles did not ameliorate the disease, but rather enhanced its severity from day 19 as shown by the significant increase in disease clinical score compared to the TNE-treated mice $(\mathrm{p}<0.05)$. These results were confirmed by the significantly greater weight loss of rSFV10-E-IFN-ß3treated mice $(\mathrm{p}<0.001)$ than of TNE-treated mice (Fig. 3). Treatment with rSFV10-E empty particles also significantly exacerbated the disease compared to TNE-treated mice $(\mathrm{p}<0.05)$, but the weight loss observed was not significant. Treatment with IFN-B-expressing particles immediately prior to and throughout the effector stage (days 5, 8, 11, 13 and 17) provided some improvement in disease clinical score. However, this improvement was significant only when

Table I. Comparison of rSFV10-E-IFN- 3 administration regimes in the treatment of EAE.

\begin{tabular}{|c|c|c|c|c|c|c|c|c|c|}
\hline \multirow[b]{2}{*}{$\begin{array}{l}\text { Treatment } \\
\text { days }\end{array}$} & \multirow[b]{2}{*}{$\begin{array}{l}\text { Experimental } \\
\text { group }\end{array}$} & \multirow[b]{2}{*}{$\begin{array}{l}\text { EAE } \\
\text { mice }\end{array}$} & \multirow[b]{2}{*}{ Mortality } & \multicolumn{2}{|c|}{ Mean $\pm \mathrm{SEM}^{\mathrm{e}}$} & \multicolumn{2}{|c|}{$\begin{array}{l}\text { p-value vs. } \\
\text { control }\end{array}$} & \multicolumn{2}{|c|}{$\begin{array}{l}\text { p-value vs. control } \\
\text { (up to day 20) }\end{array}$} \\
\hline & & & & $\begin{array}{l}\text { Maximum } \\
\text { EAE score }\end{array}$ & $\begin{array}{c}\text { Day of } \\
\text { onset }\end{array}$ & $\mathrm{TNE}^{\mathrm{a}}$ & Empty particle & $\mathrm{TNE}^{\mathrm{a}}$ & Empty particle \\
\hline \multirow{3}{*}{$\begin{array}{l}3,6,9,12,15 \\
18,21,24,27\end{array}$} & TNE & $9 / 14$ & 3 & $2.3 \pm 0.7$ & $16.6 \pm 1.09$ & NA & $0.0400^{\mathrm{b}}$ & NA & NS \\
\hline & rSFV10-E-IFN- $\beta$ & $10 / 14$ & 2 & $3.2 \pm 0.5$ & $15.2 \pm 0.93$ & $0.0400^{\mathrm{b}}$ & NS & NS & NS \\
\hline & rSFV10-E & $12 / 14$ & 0 & $2.8 \pm 0.3$ & $14.0 \pm 0.65$ & $0.0400^{\mathrm{b}}$ & NA & NS & NA \\
\hline \multirow[t]{3}{*}{$5,8,11,13,17$} & TNE & $8 / 10$ & 0 & $1.7 \pm 0.5$ & $16.6 \pm 0.84$ & NA & NS & NA & NS \\
\hline & rSFV10-E-IFN- $\beta$ & $9 / 10$ & 0 & $1.7 \pm 0.1$ & $16.2 \pm 1.29$ & NS & $0.0480^{\mathrm{b}}$ & NS & NS \\
\hline & rSFV10-E & $8 / 10$ & 0 & $2.0 \pm 0.4$ & $16.6 \pm 0.87$ & NS & NA & NS & NA \\
\hline \multirow[t]{3}{*}{$12,15,16,17$} & TNE & $7 / 10$ & 0 & $1.7 \pm 0.5$ & $13.3 \pm 0.90$ & NA & NS & NA & NS \\
\hline & rSFV10-E-IFN- $\beta$ & $8 / 10$ & 0 & $1.6 \pm 0.3$ & $13.4 \pm 1.10$ & NS & $0.0060^{c}$ & NS & NS \\
\hline & rSFV10-E & $7 / 10$ & 0 & $2.4 \pm 0.4$ & $13.6 \pm 0.78$ & NS & NA & NS & NA \\
\hline \multirow[t]{3}{*}{$5,8,11,12$} & TNE & $9 / 10$ & 0 & $1.8 \pm 0.4$ & $13.5 \pm 0.75$ & NA & $0.0008^{\mathrm{d}}$ & NA & NS \\
\hline & rSFV10-E-IFN- $\beta$ & $10 / 10$ & 0 & $1.6 \pm 0.3$ & $15.0 \pm 0.80$ & NS & $0.0003^{\mathrm{d}}$ & $0.046^{\mathrm{b}}$ & $0.0080^{\mathrm{c}}$ \\
\hline & rSFV10-E & $10 / 10$ & 0 & $2.3 \pm 0.5$ & $14.3 \pm 0.86$ & $0.0008^{\mathrm{d}}$ & NA & NS & NA \\
\hline \multirow{3}{*}{$\begin{array}{l}5,8,11,12 \\
\text { (replicate) }\end{array}$} & TNE & $10 / 12$ & 0 & $1.9 \pm 0.3$ & $15.0 \pm 0.89$ & NA & $0.0086^{\mathrm{c}}$ & NA & NS \\
\hline & rSFV10-E-IFN- $\beta$ & $10 / 12$ & 0 & $1.4 \pm 0.4$ & $16.4 \pm 1.57$ & NS & $0.0002^{\mathrm{d}}$ & $0.050^{\mathrm{b}}$ & $0.0077^{\mathrm{c}}$ \\
\hline & rSFV10-E & $9 / 12$ & 0 & $2.7 \pm 0.4$ & $13.1 \pm 0.92$ & $0.0086^{\mathrm{c}}$ & NA & NS & NA \\
\hline
\end{tabular}

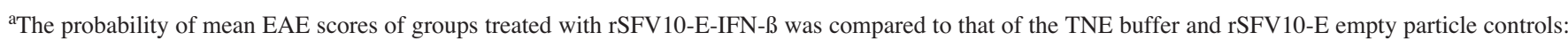
${ }^{\mathrm{b}} \mathrm{p} \leq 0.05,{ }^{\mathrm{c}} \mathrm{p}<0.01,{ }^{\mathrm{d}} \mathrm{p}<0.001 ; \mathrm{NA}$, not applicable; NS, not significant. ${ }^{\mathrm{e}}$ Standard error of the mean. 


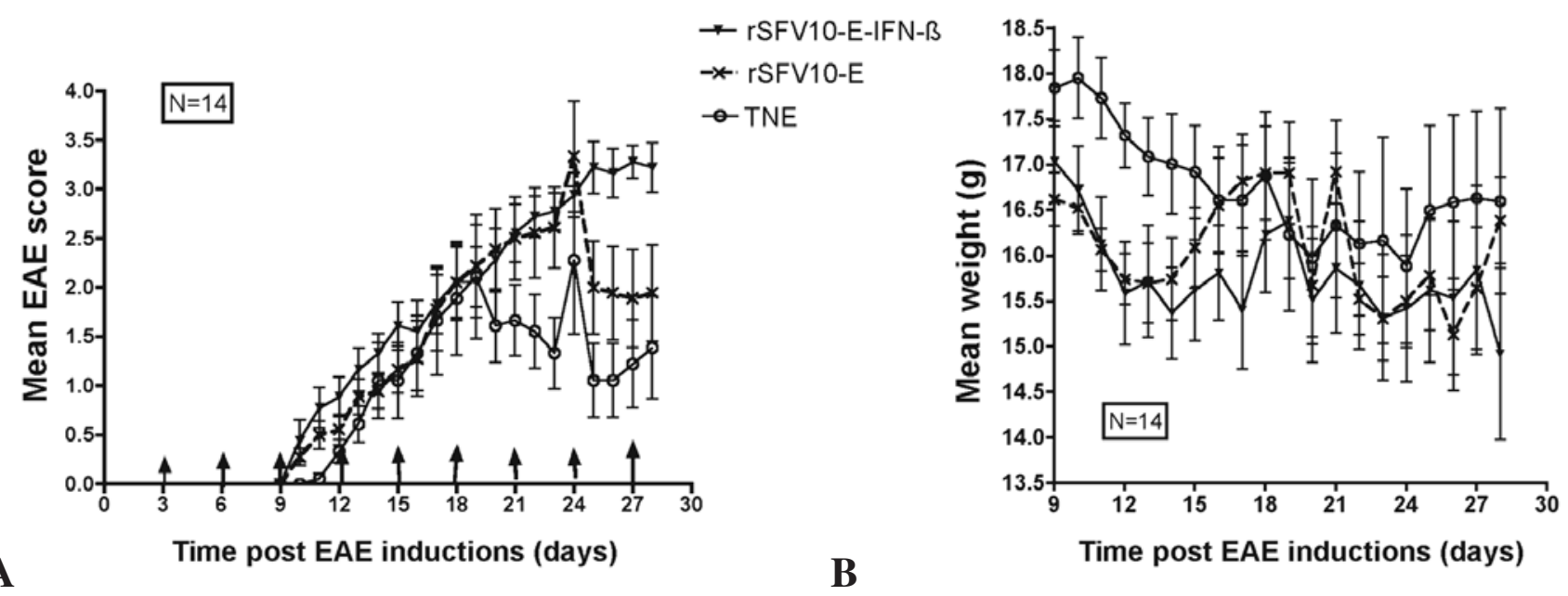

Figure 3. Effect of continuous rSFV10-E-IFN- $\beta$ treatment on the EAE disease score (A) and weight gain (B). Arrows represent treatment times. Continuous treatment exacerbated the disease from day 19 onwards. Statistical analysis was carried out using the Mann-Whitney non-parametric ranking test.
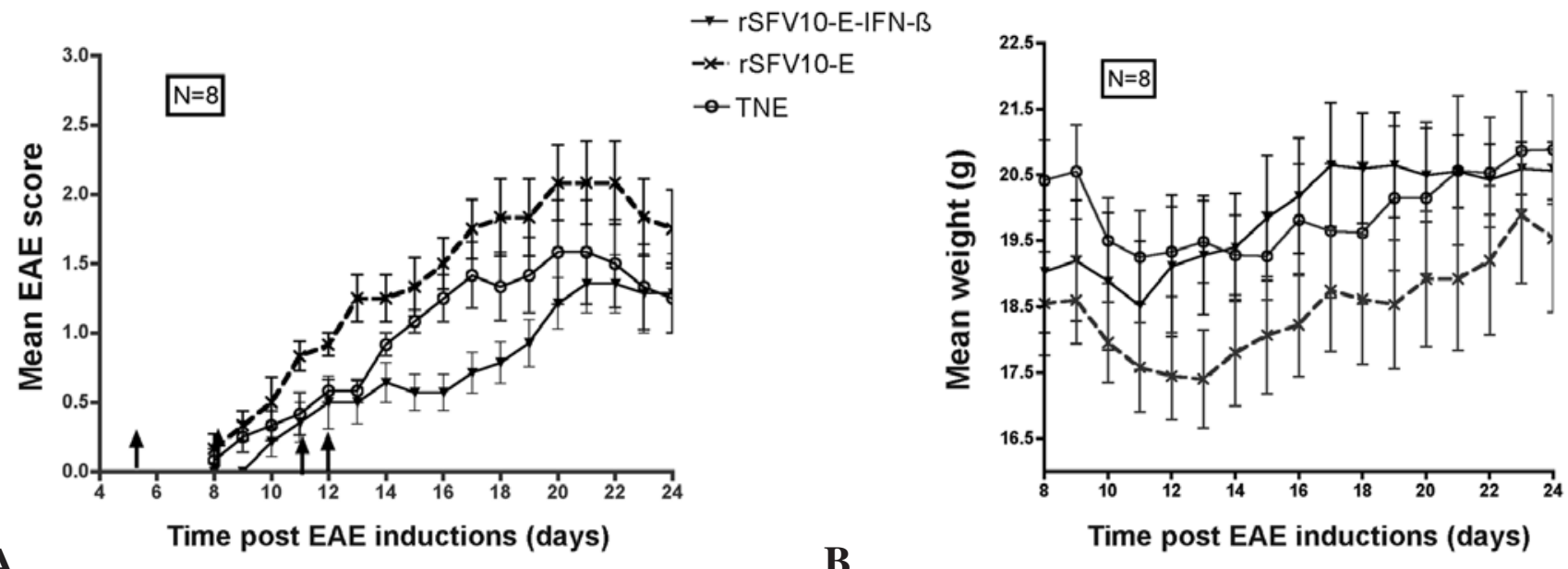

Figure 4. Effect of four rSFV10-E-IFN- $\beta$ treatments before the effector stage on EAE score (A) and weight (B). Arrows represent treatment times. Treatment with rSFV10-E-IFN- $\beta$ particles on days $5,8,11$ and 12 significantly delayed disease progression compared to the TNE- and the empty particle-treated animals up to day 20, $(\mathrm{p}<0.05)$ and $(\mathrm{p}<0.01)$ respectively (A). This was confirmed by an increase in weight compared to the controls ( $<<0.01)(B)$. Statistical analysis between the different treatment groups was carried out using the Mann-Whitney non-parametric ranking test.

compared with rSFV10-E empty particle-treated mice $(\mathrm{p}<0.05)$ and not with control TNE-treated mice. Treatment with rSFV10-E-IFN- $\beta$ particles throughout the effector stage (days $12,15,16$ and 17) prevented disease progression between days 16-26. However, again this improvement was significant only compared to rSFV10-E empty particle-treated mice $(\mathrm{p}<0.01)$. Treatment with $\mathrm{rSFV} 10-\mathrm{IFN}-\beta$ particles immediately prior to the effector stage (days 5, 8, 11 and 12) prevented disease progression between days $12-20$ as compared to the TNEtreated mice $(\mathrm{p}<0.05)$ and the $\mathrm{rSFV} 10-\mathrm{E}$ empty particle-treated mice $(\mathrm{p}<0.01)$. This was confirmed by a gradual weight gain in these mice after an initial weight loss ( $p<0.001$, Fig. 4). The rSFV10-E empty particle-treated mice showed significant exacerbation of disease clinical score as compared to the TNEtreated mice $(\mathrm{p}<0.001)$. Discontinuation of treatment led to progression of the disease, but at a gradual and less severe rate. A repeat of the experiment confirmed this result (data not shown).
Histological analysis. The correlation between the clinical and pathological scores of the mice was consistent with the more severe EAE lesions occurring in the mice with higher disease clinical scores. As expected for this EAE model, lesions were more common and severe in the spinal cord than in the brain, and lesions were invariably severe (grade 4) when both the spinal cord and brain were affected. EAE lesions were localised to white matter areas and characterised by myelin vacuolation, axonal degeneration, astrocytic proliferation and infiltrates of lymphocytes, macrophages and neutrophils.

In the continuously-treated groups, where disease severity was enhanced, examination of brain sections from mice sacrificed on day 28 showed little difference in lesion severity between rSFV10-E-IFN-ß expressing particle-, rSFV10-E empty particle- and TNE-treated mice. All mice exhibited high EAE grades and severe lesions in the brain and spinal cord. In the groups treated immediately prior to the effector stage, at which time disease progression was halted, the brain and spinal 
cord sections of mice sacrificed on days 15 and 22 showed less severe lesions in treated mice than in rSFV10-E empty particleand TNE-treated mice.

\section{Discussion}

We examined the effect of IN administration of SFV particle vectors expressing IFN- $B$ on the progression of EAE in a mouse model, showing that disease progression is inhibited only if treatment is targeted at the effector stage of the disease. Previous studies have demonstrated the inhibitory effects of IFN- $\beta$ in the treatment of $\operatorname{EAE}(25,26)$; in this study we administered IFN- $\beta$ encoded by a viral vector via the IN route. A previous study, using a retroviral vector expressing lower IFN- $\beta$ levels than the rSFV vector administered IC, also reduced clinical severity when the effector stage was targeted. This is in keeping with the results obtained here (26).

The expression and persistence of IFN- $\beta$ secreted from the vector in the CNS were examined following IN administration. This route of administration was chosen as it has been shown to be an effective non-invasive method of delivering cytokines in the treatment of EAE $(5,18,27)$. Inoculation of SFV recombinant particles by the more invasive IC route has been shown to be neurotoxic (12).

High levels of IFN- $\beta$ were found in the olfactory bulbs of rSFV10-E-IFN- $\beta$-treated mice $24 \mathrm{~h}$ after inoculation compared to the levels found in the olfactory bulbs of the TNE- and empty particle-treated controls. These levels had decreased by $48 \mathrm{~h}$ and were comparable to those measured in the olfactory bulbs of empty particle-treated animals. This confirms the findings of our previous study, which showed high level EGFP and IL-10 expression in the olfactory bulbs of mice as early as one day following IN inoculation with rSFV-EGFP or rSFVIL-10 particles. Vector RNA was only detected in the olfactory mucosa, while protein expression was detected in axons in the olfactory bulb, suggesting the viral vector replicates in the cells of the olfactory mucosa but does not enter the CNS (5). The level of IFN- $\beta$ in the remainder of the brain and spinal cord of treated mice was elevated compared to the control groups by $48 \mathrm{~h}$ post inoculation. Thus, IFN- $\beta$ persists within the CNS for two days after IN inoculation and is disseminated to the brain and spinal cord. Low levels of IFN- $B$ were also produced in the CNS tissues of empty particle-treated animals, indicating that the viral vector alone induces limited production of endogenous IFN- $\beta$ at the site of inoculation.

Continuous administration of IFN- $\beta$ throughout the induction, effector and recovery stages of EAE exacerbated disease severity. Mice showed increasing EAE scores and weight loss, correlated with the severity of the lesions at 28 days PDI. This contrasts with a previous study, which showed that daily treatment of EAE in Lewis rats with SC injections of high dose recombinant rat IFN- $\beta$ inhibits the disease, with discontinuation of treatment leading to disease worsening (28). In our previous study, we showed that treatment of EAE with rSFV-E empty vector, as opposed to vector encoding IL-10, exacerbated the disease (5). It is possible that continuous administration of rSFV10-E-IFN- $\beta$ in the present study may have led to exacerbation by the vector alone that was too severe to be countered by IFN- $\beta$ expression.

A treatment regime was therefore developed that targeted the effector stage of the disease, which fell between days 12 and 17, when the T-cell response to the encephalitogenic peptide had already developed but significant accumulation in the CNS had not yet occurred (14). Treatment of mice prior to this effector phase resulted in significant improvement in the disease. Thus this and our previous study (5) indicate that IN delivery of rSFV vectors expressing cytokines has potential as a treatment for EAE and possibly ultimately MS. However, further study is required to test their biosafety and to more fully determine host immune responses to the vector.

\section{Acknowledgements}

We thank Dorothy Mooney for assistance with the laboratory protocols and Alex Whelan-Buckley for assistance with the histopathological studies. We also thank Margaret MorrisDownes and Alan Jerusalmi for all their assistance and advice in setting up the EAE model. This work was supported by the National Neuroscience Network of Ireland, the European Union 5th Framework Programme, The Multiple Sclerosis Society of Ireland and Enterprise Ireland.

\section{References}

1. Lundstrom K: Alphavirus vectors for vaccine production and gene therapy. Expert Rev Vaccines 2: 447-459, 2003.

2. Atkins GJ, Smyth JW, Fleeton MN, Galbraith SE and Sheahan BJ: Alphaviruses and their derived vectors as anti-tumor agents. Curr Cancer Drug Targets 4: 597-607, 2004.

3. Ehrengruber MU, Renggli M, Raineteau O, Hennou S, VahaKoskela MJ, Hinkkanen AE and Lundstrom K: Semliki Forest virus A7(74) transduces hippocampal neurons and glial cells in a temperature-dependent dual manner. J Neurovirol 9: 16-28, 2003.

4. Vaha-Koskela MJ, Tuittila MT, Nygardas PT, Nyman JK, Ehrengruber MU, Renggli $\mathrm{M}$ and Hinkkanen AE: A novel neurotropic expression vector based on the avirulent A7(74) strain of Semliki Forest virus. J Neurovirol 9: 1-15, 2003.

5. Jerusalmi A, Morris-Downes MM, Sheahan BJ and Atkins GJ: Effect of intranasal administration of Semliki Forest virus recombinant particles expressing reporter and cytokine genes on the progression of experimental autoimmune encephalomyelitis. Mol Ther 8: 886-894, 2003.

6. Vähä-Koskela MJ, Kuusinen TI, Holmlund-Hampf JC, Furu PT, Heikkilä JE and Hinkkanen AE: Semliki Forest virus vectors expressing transforming growth factor beta inhibit experimental autoimmune encephalomyelitis in Balb/c mice. Biochem Biophys Res Commun 355: 776-781, 2007.

7. Balluz IM, Glasgow GM, Killen HM, Mabruk MJ, Sheahan BJ and Atkins GJ: Virulent and avirulent strains of Semliki Forest virus show similar cell tropism for the murine central nervous system but differ in the severity and rate of induction of cytolytic damage. Neuropathol Appl Neurobiol 19: 233-239, 1993.

8. Sammin DJ, Butler D, Atkins GJ and Sheahan BJ: Cell death mechanisms in the olfactory bulb of rats infected intranasally with Semliki forest virus. Neuropathol Appl Neurobiol 25: 236-243, 1999.

9. Smerdou C and Liljestrom P: Two-helper RNA system for production of recombinant Semliki Forest virus particles. J Virol 73: 1092-1098, 1999.

10. Broberg E, Setala N, Roytta M, et al: Expression of interleukin-4 but not of interleukin-10 from a replicative herpes simplex virus type 1 viral vector precludes experimental allergic encephalomyelitis. Gene Ther 8: 769-777, 2001.

11. Furlan R, Poliani PL, Marconi PC, et al: Central nervous system delivery of interleukin 4 by a nonreplicative herpes simplex type 1 viral vector ameliorates autoimmune demyelination. Human Gene Ther 9: 2605-2617, 1998.

12. Graham A, Walker R, Baird P, Hahn CN and Fazakerley JK: CNS gene therapy applications of the Semliki Forest virus 1 vector are limited by neurotoxicity. Mol Ther 13: 631-635, 2006

13. Segal BM: CNS chemokines, cytokines and dendritic cells in autoimmune demyelination. J Neurol Sci 228: 210-214, 2005. 
14. Skundric DS: Experimental models of relapsing-remitting multiple sclerosis: current concepts and perspective. Curr Neurovasc Res 2: 349-362, 2005.

15. Khoury SJ, Hancock WW and Weiner HL: Oral tolerance to myelin basic protein and natural recovery from experimental autoimmune encephalomyelitis are associated with down-regulation of inflammatory cytokines and differential upregulation of transforming growth factor beta, interleukin 4, and prostaglandin $\mathrm{E}$ expression in the brain. J Exp Med 176: 1355-1364, 1992.

16. Samoilova EB, Horton JL and Chen Y: Acceleration of experimental autoimmune encephalomyelitis in interleukin-10-deficient mice: roles of interleukin-10 in disease progression and recovery. Cell Immunol 188: 118-124, 1998.

17. Young DA, Lowe LD, Booth SS, et al: IL-4, IL-10, IL-13, and TGF-beta from an altered peptide ligand-specific Th 2 cell clone down-regulate adoptive transfer of experimental autoimmune encephalomyelitis. J Immunol 164: 3563-3572, 2000.

18. Xiao BG, Bai XF, Zhang GX and Link H: Suppression of acute and protracted-relapsing experimental allergic encephalomyelitis by nasal administration of low-dose IL-10 in rats. J Neuroimmunol 84: 230-237, 1998.

19. Xu LY, Huang YM, Yang JS, van der Meide PH, Link H and Xiao BG: Suppression of ongoing experimental allergic encephalomyelitis (EAE) in Lewis rats: synergistic effects of myelin basic protein (MBP) peptide 68-86 and IL-4. Clin Exp Immunol 120: 526-531, 2000.

20. Cua DJ, Hutchins B, La Face DM, Stohlman SA and Coffman RL: Central nervous system expression of IL-10 inhibits autoimmune encephalomyelitis. J Neuroimmunol 166: 602-608, 2001.

21. Piccirillo CA and Prud'homme GJ: Prevention of experimental allergic encephalomyelitis by intramuscular gene transfer with cytokine-encoding plasmid vectors. Hum Gene Ther 10: 1915-1922, 1999.
22. Sjoberg EM, Suomalainen M and Garoff H: A significantly improved Semliki Forest virus expression system based on translation enhancer segments from the viral capsid gene. Biotechnology 12: 1127-1131, 1994.

23. Chikkanna-Gowda CP, Sheahan BJ, Fleeton MN and Atkins GJ: Regression of mouse tumours and inhibition of metastases following administration of a Semliki Forest virus vector with enhanced expression of IL-12. Gene Ther 12: 1253-1263, 2005.

24. Barbero P, Verdun E, Bergui M, et al: High-dose, frequently administered interferon beta therapy for relapsing-remitting multiple sclerosis must be maintained over the long term: the interferon beta dose-reduction study. J Neurol Sci 222: 13-19, 2004.

25. Ruuls SR, De Labie MC, Weber KS, et al: The length of treatment determines whether IFN- $\beta$ prevents or aggravates experimental autoimmune encephalomyelitis in Lewis rats. J Immunol 157: 5721-5731, 1996.

26. Triantaphyllopoulos KA, Croxford JL, Baker D and Chernajovsky Y: Cloning and expression of murine IFN- $\beta$ and a TNF antagonist for gene therapy of experimental allergic encephalomyelitis. Gene Ther 5: 253-263, 1998.

27. Ross TM, Martinez PM, Renner JC, Thorne RG, Hanson LR and Frey WH: Intranasal administration of interferon beta bypasses the blood-brain barrier to target the central nervous system and cervical lymph nodes: a non-invasive treatment strategy for multiple sclerosis. J Neuroimmunol 152: 66-77, 2004.

28. Van der Meide PH, De Labie MC, Ruuls SR, et al: Discontinuation of treatment with IFN- $\beta$ leads to exacerbation of experimental encephalomyelitis in Lewis rats. Rapid reversal of the anti-proliferative activity of IFN- $\beta$ and excessive expansion of autoreactive $\mathrm{T}$ cells as disease promoting mechanisms. $\mathrm{J}$ Neuroimmunol 84: 14-23, 1998. 